\title{
Acute Myocardial Infarction of the Right Coronary Artery Originating From the Distal Left Circumflex Artery
}

\author{
Masaru Araki, MD; Masaharu Miyamoto, MD
}

\begin{abstract}
A case of acute myocardial infarction (AMI) of the distal left circumflex, near the origin of an aberrant right coronary artery is presented. Coronary stenting was successfully performed. According to several reports, this anomaly is a common site for coronary atherosclerosis, but this is the first report of AMI. (Circ J 2008; 72: 2092-2095)
\end{abstract}

Key Words: Acute myocardial infarction; Anomaly; Percutaneous coronary intervention; Stents

$\mathbf{T}$ he occurrence of a single, isolated coronary artery without other congenital cardiovascular abnormalities is a relatively rare anomaly and can be seen on coronary arteriography!-6 A right coronary artery (RCA) fed from the distal left circumflex artery (LCX) is especially rare-4 ${ }^{4}$ In patients with this anomaly, cardiac ischemia, including myocardial infarction (MI), is thought to be the cause of fatal hemodynamic dysfunction as a result of global ischemia?,4,5 We present a case of acute MI (AMI) caused by occlusion just at the origin of an aberrant RCA.

\section{Case Report}

A 76-year-old Japanese man came to the emergency room because of left anterior chest pain lasting $1 \mathrm{~h}$. Although he had a history of smoking (20 cigarettes/day), diabetes mellitus (hemoglobin A $1 \mathrm{c} 6.4 \%$ ), and hyperuricemia $(8.6 \mathrm{mg} / \mathrm{dl})$, he was not receiving any medication. He had no family history of ischemic heart disease. A lipid profile showed total cholesterol of $163 \mathrm{mg} / \mathrm{dl}$, triglycerides of $58 \mathrm{mg} / \mathrm{dl}$, and highdensity lipoprotein-cholesterol of $42 \mathrm{mg} / \mathrm{dl}$. In the emergency room, his blood pressure was $72 / 40 \mathrm{mmHg}$ and pulse was regular at 42 beats $/ \mathrm{min}$. Electrocardiography showed sinus bradycardia with long PR interval (260 ms), ST-segment elevation in leads II, III, aVF, and depression in leads I, aVL, $V_{2-6}$, indicative of an inferior AMI (Fig 1). After immediately starting a dopamine and nitroglycerin infusion, ventricular fibrillation (VF) occurred, which was defibrillated by DC shock (200J). After repeating VF defibrillation, recombinant tissue-type plasminogen activator (tPA) was administered intravenously at a dose of $27,500 \mathrm{IU} / \mathrm{kg}$ in order to recanalize the culprit lesion. After a lull in the VF and improvement of blood pressure via intra-aortic balloon pumping and right ventricular pacing $(60 \mathrm{ppm})$, emergency cardiac catheterization was performed. Left coronary arteriography showed 99\% stenosis of the distal LCX at the point

(Received September 3, 2007; revised manuscript received February 12, 2008; accepted March 4, 2008; released online October 14, 2008) Department of Internal Medicine, Moji Rosai Hospital, Kitakyushu, Japan

Mailing address: Masaru Araki, MD, Department of Internal Medicine, Moji Rosai Hospital, 3-1 Higashiminato-machi, Kitakyushu 801-8502, Japan._E-mail: jun1@ @ojih.rofuku.go.jp

All rights are reserved to the Japanese Circulation Society. For permissions, please e-mail: cj@j-circ.or.jp of origination of an aberrant RCA, and 58\% stenosis in the mid portion (segment 7) of the left anterior descending coronary artery (LAD) (Fig 2A). Right coronary arteriography could not be performed because there was no right coronary ostium, which was also confirmed by aortography (Fig 2B). After reopening the vessel by balloon at the site of the culprit lesion with a $2.5-\mathrm{mm}$ Crosssail (Guidant Co, Indianapolis, IN, USA), we could see for the first time the aberrant RCA crossing the crux and terminating near the right sinus of Valsalva. Coronary stenting was performed with a $3.5-\mathrm{mm}$ Duraflex stent (Avantec Vascular Co, Sunnyvale, CA, USA). The final angiographic result was excellent, with residual stenosis of 9\% (Fig 2C). Peak creatine kinase was 3,917 IU/L. At follow-up coronary angiography ( 2 weeks after onset), there was no restenosis at the site of stenting. Fractional flow reserve was measured at LAD \#7 using a 0.014-inch pressure wire (SmartMap; Volcano Co, Cordova, CA, USA) and because the result was 0.80 we instituted medical therapy until the 3 -month follow-up. Left ventriculography (LVG) showed slight hypokinesis of the inferior wall and the calculated ejection fraction was $54 \%$. During observation as an outpatient, he did not complain of angina pectoris, even during daily exercise. He received routine oral antiplatelet therapy with aspirin $(200 \mathrm{mg} /$ day $)$ plus ticlopidine $(200 \mathrm{mg} /$ day $)$. In the chronic phase (3 months after onset), coronary angiography revealed that although there was no in-stent restenosis (35\%), the stenosis of LAD \#7 (63\%) appeared to have progressed and coronary stenting was repeated with a 3.5-mm MULTI-LINK ZETA stent (Guidant). The final angiographic result was excellent, with residual stenosis of $12 \%$. No complications occurred after repeat percutaneous coronary intervention (PCI). The calculated ejection fraction by LVG was $63 \%$, which was an improvement.

\section{Discussion}

Occurrence of a single, isolated coronary artery without other congenital cardiovascular malformations is rarely seen on coronary angiography because the reported incidence is $0.024-0.066 \% !^{-4}$ It is especially rare in the case of the RCA originating from the distal LCX,4-6 an anomaly that has been classified as L-1 type in the classification of Lipton et al! Only 12 such cases have been published in the literature? but only a small number of these patients have 
I

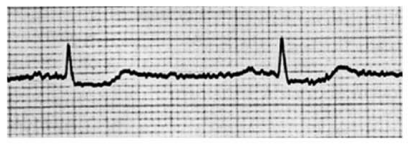

II

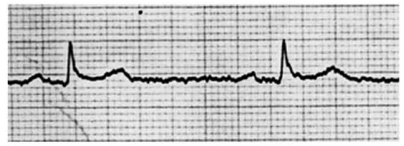

III

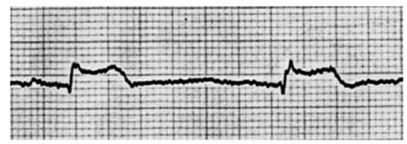

$\mathrm{aV}_{\mathrm{R}}$

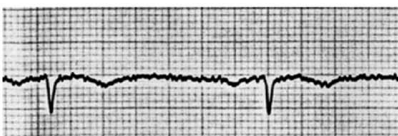

$\mathrm{aV}$

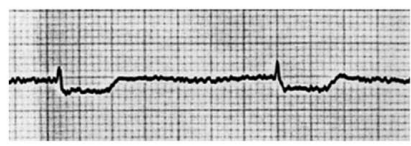

$\mathrm{aV}_{\mathrm{F}}$

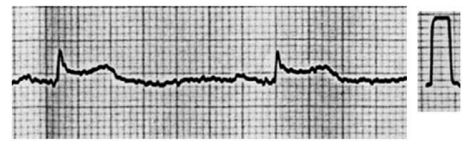

$\mathbf{V}_{1}$

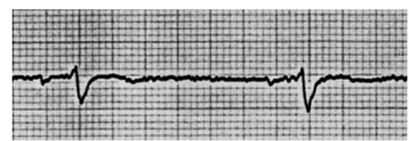

$\mathbf{V}_{2}$

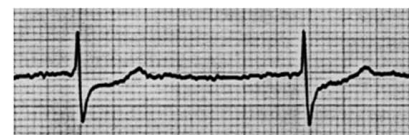

$\mathbf{V}_{3}$

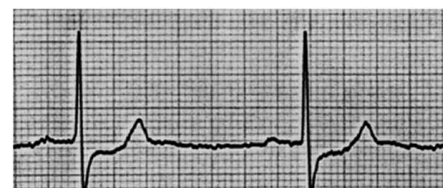

$\mathrm{V}_{4}$

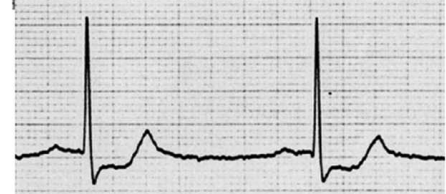

$\mathrm{V}_{5}$

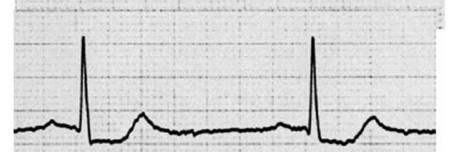

$V_{6}$

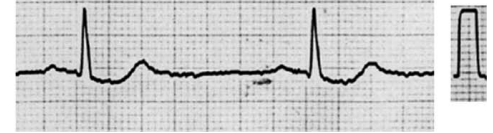

Fig 1. Electrocardiogram recorded in the emergency room shows sinus bradycardia with long PR interval $(260 \mathrm{~ms})$, ST-segment elevation in leads II, III, aVF, and depression in leads $\mathrm{I}, \mathrm{aV} \mathrm{L}, \mathrm{V}_{2-6}$, indicative of inferior acute myocardial infarction.

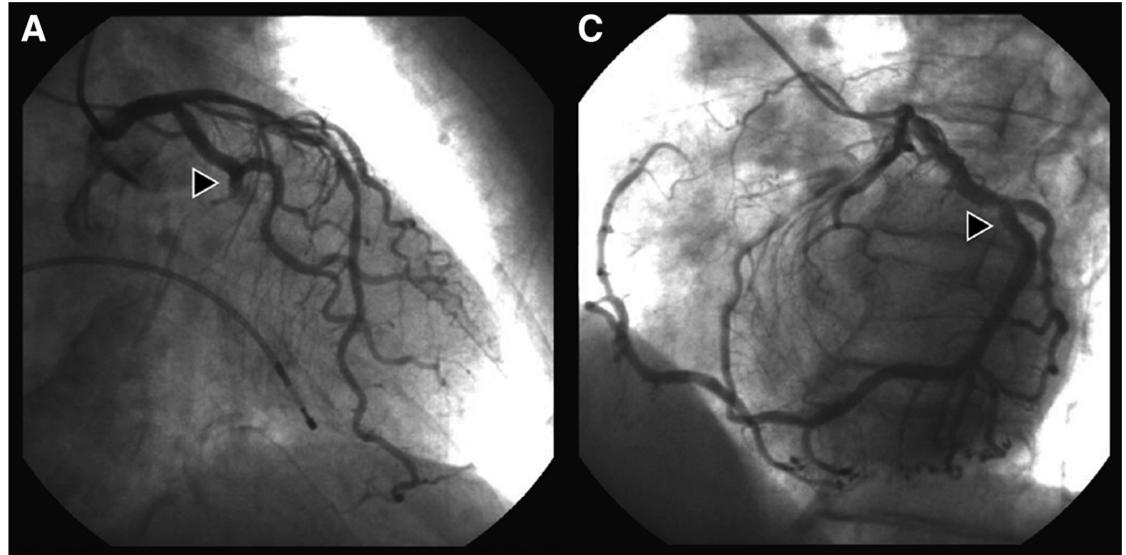

B

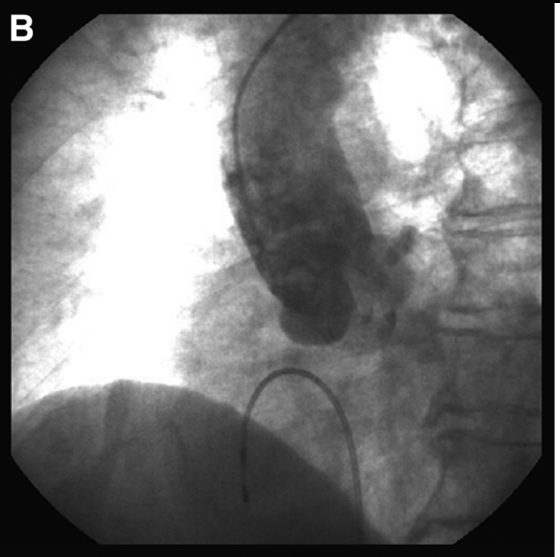

Fig 2. (A) Features of the left coronary artery in the right anterior oblique view. Culprit lesion in the left circumflex artery \#13 (arrow), at the origin of the aberrant right coronary artery (RCA), and 58\% stenosis of left anterior descending coronary artery \#7. (B) Aortography in the left anterior oblique projection showing the absence of a right coronary ostium. (C) After stenting the culprit lesion, in left anterior oblique projection we could see the aberrant RCA terminating near the right sinus of Valsalva. The final angiographic result was excellent, with residual stenosis of $9 \%$ (arrow). 
had both this anomaly and coronary heart disease requiring coronary artery bypass grafting. Currently PCI is performed more often than before and there are reports of PCI for patients with AMI of a single left coronary artery 7,8 In the present case, the culprit lesion was at the origin of an aberrant RCA feeding from the distal LCX. After several defibrillations and administration of tPA, emergency coronary angiography was performed using IABP and temporary pacing because of the cardiogenic shock. These intensive interventions were needed in this case because of the presence of an inferior AMI (electrocardiogram, LVG, bradycardia and quick recovery of hemodynamics after recanalization of aberrant RCA by PCI). It is conceivable that the pathology of an AMI caused by the occlusion of segment 1 in an anatomically normal RCA is identical to that of the present case. If the culprit lesion had been proximal to the LCX in this anomaly, the patient might have developed severe cardiogenic shock. We think the main reasons for the development of coronary atherosclerosis in the patient were his age, sex, smoking, diabetes mellitus and hyperuricemia, the common risk factors. However, we also speculate that the origin of this aberrant RCA is a common site for coronary atherosclerosis. According to 3 reports of coronary arteries that were anatomically classified as L-1 type anomaly and examined in detail by arteriography,-6 in 2 cases $^{4,5}$ there were atherosclerotic lesions just at the origin of the aberrant RCA, as in the present case. Although Sasao et al concluded there was no disturbance of blood flow in an aberrant RCA, based on measurement of coronary flow velocity from the LAD using a Doppler guidewire? we speculate that there is a predisposition to developing coronary stenosis at the origin of an aberrant RCA from the LCX. In the present case, considering there were other atherosclerotic lesions in the LAD requiring PCI, 1 of the causes of the acute coronary syndrome was the risk factors for arteriosclerosis, but we infer that turbulent coronary flow could induce unusual stress at that anatomic site? For example, a turbulent flow pattern at branch points, bifurcations and anastomoses of coronary arteries is considered to be a local biomechanical risk factor for atherosclerosis,-11 including low or reversed wall shear stress. Unfortunately, we could not directly measure the coronary flow pattern at the culprit lesion in the present case, but the anatomical characteristics and past case reports suggest that this is a common site of developing atherosclerotic plaque.

The present patient experienced AMI caused by occlusion of the origin of an aberrant RCA from the distal LCX, a single isolated coronary artery of type L- 1 , which is very rarely seen, and we speculate that the anatomy of the site of origin favored the development of atherosclerosis.

\section{References}

1. Lipton MJ, Barry WH, Obrez I, Silverman JF, Wexler L. Isolated single coronary artery: Diagnosis, angiographic classification, and clinical significance. Radiology 1979; 130: 39-47.

2. Desmet W, Vanhaecke J, Vrolix M, Van de Werf F, Piessens J, Willems J, et al. Isolated single coronary artery: A review of 50,000 consecutive coronary angiographies. Eur Heart J 1992; 13: 1637 1640.

3. Yamanaka O, Hobbs RE. Coronary artery anomalies in 126,595 patients undergoing coronary arteriography. Cathet Cardiovasc Diagn 1990; 21: 28-40.

4. Chou LP, Kao C, Lee MC, Lin SL. Right coronary artery originating from distal left circumflex artery in a patient with an unusual type of isolated single coronary artery. Jpn Heart J 2004; 45: 337-342.

5. Vrolix MC, Geboers M, Sionis D, De Geest H, Van de Werf F. Right coronary artery originating from distal left circumflex: An unusual feature of single coronary artery. Eur Heart J 1991; 12: 746-747.

6. Turhan H, Duru E, Yetkin E, Atak R, Senen K. Right coronary artery originating from distal left circumflex: An extremely rare variety of single coronary artery. Int J Cardiol 2003; 88: 309-311.

7. Sasao H, Hasegawa T, Endo A, Fujiwara T, Kikuchi Y, Oimatsu H, et al. Coronary stent implantation in patients with a single coronary artery: A report of 3 cases. Angiology 2003; 54: 131-135.

8. Takano M, Seimiya K, Yokoyama S, Okamatsu K, Ishibashi F, Uemura R, et al. Unique single coronary artery with acute myocardial infarction: Observation of the culprit lesion by intravascular ultrasound and coronary angioscopy. Jpn Heart J 2003; 44: 271-276.

9. Akasaka T, Karino T. Flow patterns and spatial distribution of atherosclerotic lesions in human coronary arteries. Circ Res 1990; 66: 10451066.

10. Feldman CL, Stone PH. Intravascular hemodynamic factors responsible for progression of coronary atherosclerosis and development of vulnerable plaque. Curr Opin Cardiol 2000; 17: 430-440.

11. Freshwater IJ, Morsi IS, Lai T. The effect of angle on wall shear stresses in a LIMA to LAD anastomosis: Numerical modelling of pulsatile flow. Proc Inst Mech Eng [H]. 2006; 220: 743-757.

12. Tavernarakis A, Voudris V, Ifantis G, Tsaganos N. Anomalous origin of the right coronary artery arising from the circumflex artery. Clin Cardiol 1986; 9: 230-232.

13. Sheth M, Dovnarsky M, Cha SD, Kini P, Maranhao V. Single coro-

Table 1 Reports of the L-1 Type Anomaly

\begin{tabular}{cccccc}
\hline \hline Case & Reference & Age $($ years)/sex & AP & Angiography & Therapy \\
\hline 1 & Lipton $(1979)^{1}$ & $30 / M$ & Atypical & No significant stenosis & ND \\
2 & Tavernarakis $(1986)$ & $57 / M$ & + & LAD occlusion & ND \\
3 & Sheth $(1988)$ & $60 / M$ & Atypical & No significant stenosis & None \\
4 & Vrolix $(1991)^{5}$ & $51 / M$ & + & LCX, 80\% stenosis & Surgical \\
5 & Desmet $(1992)^{2}$ & $52 / M$ & Atypical & No significant stenosis & ND \\
6 & Desmet $(1992)^{2}$ & $53 / M$ & $N D$ & Atherosclerosis & Surgical \\
7 & Desmet $(1992)^{2}$ & $45 / M$ & $N D$ & Atherosclerosis & Surgical \\
8 & Desmet $(1992)^{2}$ & $41 / M$ & $N D$ & Atherosclerosis & Medical \\
9 & Desmet $(1992)^{2}$ & $64 / M$ & ND & Atherosclerosis & Medical \\
10 & Desmet $(1992)^{2}$ & $55 / M$ & ND & Atherosclerosis & Medical \\
11 & Desmet $(1992)^{2}$ & $51 / M$ & ND & Atherosclerosis & Surgical \\
12 & Turhan $(2002)^{6}$ & $52 / M$ & Atypical & No significant stenosis & None \\
13 & Chou $(2004)^{4}$ & $42 / M$ & + & LCX, 40\% stenosis & None \\
14 & Yoshimoto $(2004)$ & $63 / M$ & Atypical & No significant stenosis & None \\
15 & Present case $(2004)$ & $76 / M$ & + & LCX occlusion & PCI \\
\hline
\end{tabular}

$A P$, angina pectoris; ND, not discussed; LAD, left anterior descending artery; LCX, left circumflex artery; PCI, percutaneous coronary intervention. 
nary artery: Right coronary artery originating from distal left circumflex. Cathet Cardiovasc Diagn 1988; 14: 180-181.

14. Yoshimoto S, Hirooka K, Irino H, Abe H, Yasuoka Y, Yamamoto H, et al. Anomalous right coronary artery originating from the distal left circumflex artery: Single coronary artery with choronic atrial fibrillation. Jpn Heart J 2004; 45: 679-683.

\section{Appendix 1}

Reports of the L-1 type anomaly! Cases 6-11 do not have detailed arteriography findings. There was significant stenosis in cases $2,4,13$ and the present case. The origin of the aberrant RCA was seen in cases 4,13 and the present case (Table 1). 